\title{
Aspetos cronobiológicos e do sono associados ao neurodesenvolvimento e desempenho escolar em idade pediátrica
}

\section{Chronobiological and sleep related aspects associated to neurodevelopment and academic performance in pediatric age}

\author{
Miguel Meira e Cruz, Unidade de Sono, Centro Cardiovascular da Universidade de Lisboa \\ Faculdade de Medicina de Lisboa, Portugal (mcruz@medicina.ulisboa.pt) \\ Fernanda Dubourg, Unidade de Saúde da Criança e Adolescente, Ambulatório de Neurologia Pediátrica, \\ Complexo Hospitalar Universitário Professor Edgard Santos - Universidade Federal da Bahia, Salvador, Brasil \\ (corfernanda@hotmail.com)
}

Resumo: Os ritmos biológicos, e o sono enquanto seu representante de destaque, são fatores biofuncionais relevantes no desenvolvimento neuro-cognitivo e comportamental, com particular interesse no domínio pediátrico. Aspetos concretos, associados à homeostasia do sistema nervoso central e de funções superiores como o equilíbrio socio-afetivo, o juízo crítico, a consolidação de memórias e a capacidade de aprendizagem, relacionam-se frequentemente, nestas fases da vida, com a estabilidade do sistema temporal circadiano e com a individualidade temporal definida pelo cronotipo. $\mathrm{O}$ sono inadequado ou insuficiente tem, por outro lado, um impacto negativo em diversos parâmetros biopsicossociais que interagem com esses domínios e que condicionam o normal desenvolvimento traduzido em alterações no desempenho neuro-cognitivo e comportamental. Esta revisão incide sobre os aspetos fundamentais da cronobiologia e da fisiologia do sono aplicados ao neurodesenvolvimento e sua aplicabilidade no contexto educacional em relação com o desempenho escolar.

Palavras-Chave: neurodesenvolvimento, performance académica, ritmos circadianos, sono

\begin{abstract}
Biological rhythms and sleep as their main representative, are relevant biofunctional factors to the neuro-cognitive and behavioral development with particular interest in pediatric domain. Some aspects associated to central nervous system and higher function homeostasis, like social-affective equilibrium, critical judgement and memories consolidations and learning, have, in this phase of life, a relationship with the circadian timing system stability and with chronotype. On the other hand, inadequate or insufficient sleep has a negative impact in several biopsychosocial parameters interacting with these domains and conditioning the normal development causing changes in neuro-cognitive and behavioral performance. This review looked for the fundamental aspects of chronobiology and sleep physiology applied to neurodevelopment and its impact in the educational context regarding academic performance.
\end{abstract}

Keywords: neurodevelopment, academic performance, circadian rhythms, sleep 


\section{Introdução}

A vida na Terra é previsível. A rotação do planeta num eixo central condiciona um movimento continuo com um período de aproximadamente 24h. Este movimento que, pela coincidência temporal e periódica por exposição ao sol, determina uma fase de claridade e outra de escuridão (o dia e a noite solares) tem sido, ao longo da evolução da espécie, um determinante para adaptações moleculares, celulares, orgânicas e sistémicas, possibilitando ao corpo humano, dos mamíferos e de virtualmente toda a matéria viva, a capacidade de antecipação, com vantagens para o sucesso (Patke et al, 2020). Para que essa capacidade antecipatória fosse efetiva, a biologia construiu, nos seres vivos, um sistema temporal complexo multimodal no que respeita a parâmetros como frequência, período, amplitude, máximos e mínimos definidos para cada variável de controlo (Refinetti et al, 2012). Dos vários ritmos biológicos evidenciados no organismo humano, um tipo tem ganho particular destaque, pela relevância fisiológica no controlo diário de diversas funções, entre as quais está o ciclo vigília-sono. Porque se repetem em cada cerca de um dia, estes ritmos foram designados de circadianos (do latim=circa diem, ou cerca das $24 \mathrm{~h}$ do dia solar) e são regulados por um sistema composto por um relógio central, $\mathrm{o}$ núcleo supraquiasmático do hipotálamo, e por osciladores periféricos, sincronizados por este (Koronowski et al, 2021). Este sistema toma, no seu conjunto, o nome de Sistema Temporal Circadiano e o seu conhecimento representa um importante avanço para a biologia e medicina contemporâneas (Allada et al, 2021).

\section{Ritmos Biológicos, Sistema Temporal Circadiano e Cronotipo}

No ambiente materno, alguns ritmos são já evidentes. A sincronização é, no entanto, neste período da vida, condicionada pela secreção de melatonina materna. Entende-se, pois, neste contexto, que alguns dos acontecimentos perinatais sejam guiados por eles (Bates et al, 2020). Por exemplo, existem mais partos durante a madrugada e quando estes ocorrem em horas noturnas, são por norma mais rá- pidos. O movimento fetal é mais frequente durante a noite, sincronizado com o início do sono da mãe, e a respiração acelera no período noturno, após ingestão de alimento. A hemodinâmica, os níveis tensionais, a secreção de cortisol e progesterona também espelham esta natureza circadiana da vida fetal, e que persiste após o nascimento. Por outro lado, a cronodisrupção materna tem uma influência não negligenciável no desenvolvimento patológico do feto (Hsu et al, 2020; Loy et al, 2020).

Apesar disto, algumas variáveis fisiológicas adquirem uma ritmicidade circadiana só após o parto, sugerindo que o contacto com osciladores externos, nomeadamente a alternância entre $o$ dia e a noite são fundamentais para algumas funções.

$\mathrm{O}$ tempo não passa por todos da mesma forma. A maioria dos estudos envolvendo a individualidade temporal interna são controversos no que respeita à definição de cronotipo, já que usam como indicador, a preferência horária (manhã versus tarde/ noite) das atividades, em vez do comportamento de sono (ponto médio do sono) ou de marcadores biológicos do relógio (início da secreção de melatonina) (Bauducco et al. 2020). É conhecida a relação entre a fase da temperatura corporal e a melatonina, e de ambas com o cronotipo individual. Em crianças com o cronotipo matutino, encontramos uma maior amplitude na variação, quer da temperatura corporal quer do perfil de secreção da melatonina. Também a fase descendente da temperatura ocorre mais cedo nos matutinos e a melatonina tem um início de produção mais precoce. Este tema complexo, que tem recebido ainda menos atenção no contexto pediátrico tem implicações importantes já que, por exemplo, crianças matutinas têm mais dificuldade em adormecer e de despertar por si. Por outro lado, o cronotipo vespertino neste grupo etário, associa-se a uma maior resistência na hora de deitar, assim como a uma maior probabilidade de despertar com alterações no humor, que invariavelmente condicionam conflitos com os pais e/ou educadores (Jafar et al, 2017). Numa perspetiva clínica, o cronotipo tornase também um aliado precioso na condução terapêutica, orientando-a e viabilizando a sua personalização, com resultados potencialmente mais efetivos. 


\section{Fundamentos sobre o sono em idade pediátrica}

A mais óbvia manifestação de um ritmo circadiano é, no organismo humano, o ciclo vigília-sono. $\mathrm{O}$ sono noturno, em particular, é tido como fundamental na nossa espécie, já que dele dependem inúmeras ações fisiológicas e comportamentos que se associam a uma vida saudável e duradora. Na sua ausência, ou no caso de um sono perturbado, voluntariamente ou por doença, existe frequentemente disfunção a vários níveis com impacto a nível cardiometabólico, cognitivo e comportamental. Do ponto de vista da ontogenética, o sono parece ter um papel essencial no desenvolvimento do sistema nervoso, em particular do cérebro. Sabe-se que recém-nascidos a termo dormem 16 a 18 horas por dia e $50 \%$ desse tempo em sono REM. Os prematuros passam ainda mais tempo dormindo com até $80 \%$ do tempo em REM. Esse maior tempo de sono das crianças nos primeiros anos de vida parece refletir o papel fundamental do sono, e em especial do sono REM, no desenvolvimento cerebral na infância (Challamell et al, 1992). O sono em idade pediátrica assume, de facto, um particular interesse biológico, já que influencia processos de neurodesenvolvimneto e de crescimento indispensáveis nestas fases da vida. É organizado desde cedo, ainda em ambiente intra-uterino. Ao fim dos primeiros 6 meses de vida, o recém-nascido terá adquirido a expressão completa do sistema circadiano no sono, podendo este processo ser atrasado na prematuridade (De Beritto et al, 2020). Ao contrário do que sucede com a componente homeostática de regulação do sono, pela qual a predisposição para o sono aumenta à medida que aumenta o tempo de vigília, a componente circadiana tem uma tendência cíclica que é independente do tempo de vigília anterior. Isto é, o ritmo circadiano da predisposição ao sono é um fator determinante para que, por exemplo, a criança ou o adolescente, possam ter dificuldade em iniciar o sono noturno dentro do horário desejado, ainda que tenham uma divida de sono anterior por terem dormido pouco nas noites que antecederam. Desta forma, algumas crianças podem apresentar um quadro de sonolência exacerbada durante as horas destinadas à escola ou ao estudo, sendo, por es- te motivo, possível que uma criança com um horário de sono idealizado para as $21 \mathrm{~h}$ se mantenha desperto até às $00 \mathrm{~h}$.

$\mathrm{Na}$ idade escolar, aspetos sociais e comportamentais associados a um horário mais tardio em ir para a cama durante o final de semana, assim como um despertar mais tardio, favorecem o atraso de fase fisiológico e pode gerar dificuldades também no despertar a horas na segunda feira subsequente. Já na pré-adolescência o sono vai gradualmente atrasando em relação com a noite biológica, resultando num atraso mais marcado na propensão para o sono (Bathory et al, 2017).

\section{Ritmos circadianos, sono e Emoções}

Um conjunto importante de estudos tem estabelecido relações entre o sono inadequado e alterações na regulação emocional e investigações recentes sugerem uma partilha de fatores genéticos entre distúrbios do sono e mecanismos associados à psicopatologia na criança, nomeadamente à impulsividade e à ira/frustração (Miadich et al. 2020). Kym e colaboradores avaliaram recentemente a relação entre 0 uso de smartphones numa população de crianças com idades entre os 5 e os 8 anos, consolidando a noção de que o uso destes dispositivos afeta negativamente, quer a duração, quer a qualidade do sono (Kim et al. 2020). Desvios comuns ao desenvolvimento normal ocorrem nesta fase da vida, determinando também diferentes cuidados. Crianças com Perturbação de Hiperatividade e Défice de Atenção (PHDA) são mais sensíveis à perturbação do sono. Na realidade, a PHDA afeta de forma importante, quer a criança com a doença, quer a família, pelo que tem sido defendida a aplicação de estratégias comportamentais para a criança com o diagnóstico e estratégias de intervenção sobre a saúde mental para o agregado familiar (Martin et al, 2021). Um estudo recente mostrou inclusivamente que, quando recebem tratamento adequado, estas crianças corrigem quer os défices emocionais, quer os problemas de sono, reforçando a relação entre ambos os fatores (Sanabra et al, 2021). Uma das questões mais debatidas em medicina do sono pediátrica é a oportunidade e vantagem das sestas para o desenvolvimento 
adequado. Os resultados de Bacaro et al, num estudo recente, que avaliou a associação entre os padrões de sono diurno e o perfil emocional em crianças e bebés, revelaram que o sono diurno está, nas crianças mais novas, positivamente relacionado com um desenvolvimento saudável, no espectro cognitivo-emocional (Bacaro et al, 2020). Estes resultados são a favor da defesa da sesta, pelo menos até aos 5 anos de idade (Staton et al, 2019).

\section{Sono, Aprendizagem, Memória e Atenção}

Diversos trabalhos têm demonstrado também que a consolidação de memórias declarativas, ou episódicas, motoras, emocionais, sensoriais e olfativas ocorre preferencialmente durante o sono (MacDonald et al, 2021). O sono após a aprendizagem de uma nova tarefa, de uma habilidade nova, ou da aquisição de informação, facilita a retenção dessa aprendizagem (Acosta et al, 2019). Os mecanismos parecem estar relacionados com o sono de ondas lentas e, neste contexto, têm sido desenvolvidas algumas técnicas de neuroestimulação que parecem favorecer a consolidação de memórias por via deste papel mediador que o sono profundo assume no processo (Malkani et al, 2020). Em contraste, o sono inadequado, pode ter interferência negativa nesta capacidade. Em simultâneo, por via da interação com o sistema neuroimune, e com o eixo hormona de crescimento/fator de crescimento similar a insulina, o sono perturbado pode resultar na perda de capacidade de retenção de memórias, assim como em efeitos deletérios em vários outcomes relacionados com a idade (Chennaoui et al, 2020). A insónia na idade adulta foi associada também a alterações cognitivas durante 0 dia (Brownlow et al, 2020). Dadas as vias fisiopatológicas, provavelmente comuns na idade pediátrica e sendo a insónia frequentemente esquecida neste grupo etário, é provavelmente importante, reconhecer a condição para um prognóstico favorável em todas as dimensões, clínicas e terapêuticas (Himelfarb et al 2021; Ekambaram et al 2021). É igualmente importante ter em mente que outras alterações do sono com impacto na aprendizagem, memória e cognição podem frequentemente afetar crianças e adolescentes de uma forma mais grave do que o fazem na idade adulta. Nomeadamente as perturbações respiratórias devem, pela sua prevalência e sig- nificado, ser contempladas na avaliação em idades precoces, sobretudo pelo potencial impacto na função cognitiva, uma vez que influenciam as estruturas plásticas do cérebro e podem alterar o desenvolvimento neuro psíquico (Lo Blue et al. 2020). Mesmo o ressonar persistente, sem apneias, parece ter, nas crianças em idade escolar, um impacto negativo, sobretudo no contexto neurocomportamental (Hangstrom et al, 2020). Por outro lado, alterações do neurodesenvolvimento que cursem com malformações craniofaciais, como as que surgem por exemplo na Síndrome de Down, aumentam o risco de perturbação respiratória durante 0 sono (Chawla et al, 2020), contribuindo para um ciclo vicioso em que o sono inadequado aumenta o risco de alterações cognitivas, contribuindo estas também, muitas vezes para que o ciclo perpetue.

\section{Ritmos, Sono e Performance Escolar}

Uma das principais formas de manifestação clínica do sono inadequado é a performance escolar ou académica diminuída e, apesar da maioria dos estudos ter foco na adolescência, alguns estudos corroboram que a importância do sono no sucesso escolar existe já em idades precoces. Num artigo recente, Sun e colaboradores (Sun et al, 2019) mostraram uma relação de uma performance mais limitada com o aumento das diferenças de sono da semana para o fim de semana (um indicador de sono inadequado). Os autores deste estudo mostraram um risco de suicídio aumentado na mesma população, indiciando uma relação da performance académica com a saúde emocional. Rajaei et al, mostraram também que o processamento sensorial e a qualidade do sono afetam a performance de crianças na escola primária (Rajaei et al, 2020). As disritmias circadianas e as perturbações do sono são, neste contexto, provavelmente mais relevantes em populações com necessidades especiais, por exemplo aquelas com perturbação do espectro do autismo (Yavuz-Kodat et al, 2020) e com PHDA (Dimakos et al, 2021). De resto, a cronoterapia, por exemplo com recurso a melatonina, tem um efeito positivo nestes pacientes embora aspetos metodológicos sobre a forma e dose a considerar se mantenham controversos (RzepkaMingut et al, 2020). A relação do sono com a performance determina que sejam também ponderados os horários escolares. Neste sentido, vários estudos têm de- 
monstrado que o horário mais tardio no início da escola se relaciona com uma melhor qualidade de sono e com índices mais elevados de sucesso escolar (Alfonsi et al, 2020). Ruiz-Herrera e colaboradores estudaram a relação entre sono, performance académica e função cognitiva em crianças com PHDA, mostrando que existem preditores polissonográficos para a diminuição da função cognitiva e do rendimento escolar nesta população (Ruiz-Herrera et al, 2021). Esta interação de fatores tem um reflexo clínico-laboratorial importante e parece constituir um mecanismo a ser considerado, também nos adolescentes universitários com normal desenvolvimento (Suardiaz-Muro et al, 2020).

\section{Conclusão}

A sincronização rítmica condicionada pelos relógios biológicos que compõe o sistema circadiano é fundamental para o desenvolvimento normal, adquirindo especial relevo nos grupos de idade mais jovens. Ao ritmo vigília-sono, em particular, deve ser dada, na criança e no adolescente, uma atenção especial, já que são frequentes os fatores que podem perturbar a harmonia deste ciclo, com compromisso de funções indispensáveis ao normal processo de maturação e desenvolvimento emocional, cognitivo e comportamental com reflexo no rendimento e no sucesso escolar.

\section{Referências}

Acosta, M. T. (2019). Sueño, memoria y aprendizaje [Sleep, memory and learning]. Medicina (Buenos Aires), 79 (Suppl 3), 29-32. https://www.medicinabuenosaires.com/revistas/vol79-19/s3/s3-Acosta.pdf

Alfonsi, V., Palmizio, R., Rubino, A., Scarpelli, S., Gorgoni, M., D'Atri A., Pazzaglia, M., Ferrara, M., Giuliano, S., \& De Gennaro, L. (2020). The Association Between School Start Time and Sleep Duration, Sustained Attention, and Academic Performance. Nature and Science of Sleep, 12, 1161-1172. https:// doi.org/10.2147/NSS.S273875

Allada, R., \& Bass, J. (2021). Circadian Mechanisms in Medicine. New England Journal of Medicine, 384(6), 550-561. https://doi.org/10.1056/NEJMra1802337

Bacaro, V., Feige, B., Benz, F., Johann, A. F., De Bartolo, P., Devoto, A., Lombardo, C., et al. (2020). The Association between Diurnal Sleep Patterns and Emotions in Infants and Toddlers Attending Nursery. Brain Sciences, 10(11), 891. MDPI AG. http://dx.doi.org/10.3390/brainsci10110891

Bates, K., \& Herzog, E. D. (2020). Maternal-Fetal Circadian Communication During Pregnancy. Frontiers in endocrinology, 11, 198. https://doi.org/10.3389/fendo.2020.00198

Bathory, E., \& Tomopoulos, S. (2017). Sleep Regulation, Physiology and Development, Sleep Duration and Patterns, and Sleep Hygiene in Infants, Toddlers, and Preschool-Age Children. Current problems in pediatric and adolescent health care, 47(2), 29-42. https://doi.org/10.1016/j.cppeds.2016.12.001

Bauducco, S., Richardson, C., \& Gradisar, M. (2020). Chronotype, circadian rhythms and mood. Current opinion in psychology, 34, 77-83. https://doi.org/10.1016/j.copsyc.2019.09.002 
Brownlow, J. A., Miller, K. E., \& Gehrman, P. R. (2020). Insomnia and Cognitive Performance. Sleep medicine clinics, 15(1), 71-76. https://doi.org/10.1016/j.jsmc.2019.10.002

Challamel, M. J. (1992). Fonctions du sommeil paradoxal et ontogenèse [Functions of paradoxical sleep and ontogenesis]. Neurophysiologie clinique = Clinical neurophysiology, 22(2), 117-132. https:// doi.org/10.1016/s0987-7053(05)80749-x

Chawla, J. K., Burgess S., \& Heussler, H. (2020). The impact of sleep problems on functional and cognitive outcomes in children with Down syndrome: a review of the literature. Journal of Clinical Sleep Medicine, 16(10), 1785-1795. https://doi.org/10.5664/jcsm.8630

Chennaoui, M., Léger, D., \& Gomez-Merino, D. (2020). Sleep and the GH/IGF-1 axis: Consequences and countermeasures of sleep loss/disorders. Sleep Medicine Reviews, 49, 101223. https://doi.org/10.1016/ j.smrv.2019.101223

De Beritto, T. V. (2020). Newborn Sleep: Patterns, Interventions, and Outcomes. Pediatric annals, 49(2), e82e87. https://doi.org/10.3928/19382359-20200122-01

Dimakos, J., Gauthier-Gagné, G., Lin, L., Scholes, S., \& Gruber, R. (2021). The Associations Between Sleep and Externalizing and Internalizing Problems in Children and Adolescents with Attention-Deficit/ Hyperactivity Disorder: Empirical Findings, Clinical Implications, and Future Research Directions. Child and adolescent psychiatric clinics of North America, 30(1), 175-193. https://doi.org/10.1016/ j.chc.2020.08.001

Ekambaram, V., \& Owens, J. (2021). Medications Used for Pediatric Insomnia. Child and adolescent psychiatric clinics of North America, 30(1), 85-99. https://doi.org/10.1016/j.chc.2020.09.001

Hagström, K., Saarenpää-Heikkilä, O., Himanen, S. L., Lampinlampi, A. M., \& Rantanen, K. (2020). Neurobehavioral Outcomes in School-Aged Children with Primary Snoring. Archives of clinical neuropsychology: the official journal of the National Academy of Neuropsychologists, 35(4), 401-412. https:// doi.org/10.1093/arclin/acz053

Himelfarb, M., \& Shatkin, J. P. (2021). Pediatric Insomnia. Child and adolescent psychiatric clinics of North America, 30(1), 117-129. https://doi.org/10.1016/j.chc.2020.08.004

Hsu, C.-N., \& Tain, Y.-L. (2020). Light and Circadian Signaling Pathway in Pregnancy: Programming of Adult Health and Disease. International Journal of Molecular Sciences, 21(6), 2232. MDPI AG. Retrieved from http://dx.doi.org/10.3390/ijms21062232

Jafar, N. K., Tham, E. K., Eng, D. Z., Goh, D. Y., Teoh, O. H., Lee, Y. S., Shek, L. P., Yap, F., Chong, Y. S., Meaney, M. J., Gooley, J. J., Broekman, B. F., \& Gusto Study Group (2017). The association between chronotype and sleep problems in preschool children. Sleep medicine, 30, 240-244. https:// doi.org/10.1016/j.sleep.2016.11.015

Kim, S. Y., Han, S., Park, E. J., Yoo, H. J., Park, D., Suh, S., \& Shin, Y. M. (2020) The relationship between smartphone overuse and sleep in younger children: a prospective cohort study. Journal of Clinical Sleep Medicine, 16(7), 1133-1139. https://doi.org/10.5664/jcsm.8446

Koronowski, K. B., \& Sassone-Corsi, P. (2021). Communicating clocks shape circadian homeostasis. Science, 371(6530), eabd0951. https://doi.org/10.1126/science.abd0951

Lo Bue, A., Salvaggio, A., \& Insalaco, G. (2020). Obstructive sleep apnea in developmental age. A narrative review. European journal of pediatrics, 179(3), 357-365. https://doi.org/10.1007/s00431-019-03557-8

Loy, S. L., Loo, R. S. X., Godfrey, K. M., Chong, Y.-S., Shek, L.P.-C., Tan, K. H., Chong, M. F.-F., Chan, J. K. Y., \& Yap, F. (2020). Chrononutrition during Pregnancy: A Review on Maternal Night-Time Eating. $\mathrm{Nu}$ trients, 12(9), 2783. https://doi.org/10.3390/nu12092783 
MacDonald, K. J., \& Cote, K. A. (2021). Contributions of post-learning REM and NREM sleep to memory retrieval. Sleep Medicine Reviews, 59, 101453. https://doi.org/10.1016/j.smrv.2021.101453

Malkani, R. G., \& Zee, P. C. (2020). Brain Stimulation for Improving Sleep and Memory. Sleep medicine clinics, 15(1), 101-115. https://doi.org/10.1016/j.jsmc.2019.11.002

Martin, C. A., Papadopoulos, N., Rinehart, N., \& Sciberras, E. (2019). Associations Between Child Sleep Problems and Maternal Mental Health in Children with ADHD. Behavioral Sleep Medicine, 19(1), 12-25. https://doi.org/10.1080/15402002.2019.1696346

Miadich, S. A., Shrewsbury, A. M., Doane, L. D., Davis, M. C., Clifford, S., \& Lemery-Chalfant, K. (2020). Children's sleep, impulsivity, and anger: shared genetic etiology and implications for developmental psychopathology. Journal of Child Psychology and Psychiatry (JCPP), 61(10), 1070-1079. https:// doi.org/10.1111/jcpp. 13328

Patke, A., Young, M.W., \& Axelrod, S. (2020). Molecular mechanisms and physiological importance of circadian rhythms. Nature Reviews Molecular Cell Biology, 21, 67-84. https://doi.org/10.1038/s41580-019-0179-2

Rajaei, S., Kalantari, M., Pashazadeh Azari, Z., Tabatabaee, S. M., \& Dunn, W. (2020). Sensory Processing Patterns and Sleep Quality in Primary School Children. Iranian journal of child neurology, 14(3), 57-68. https://www.ncbi.nlm.nih.gov/pmc/articles/PMC7468082/

Refinetti, R. (2012). Integration of biological clocks and rhythms. Comprehensive Physiology, 2(2), 1213-39. https://doi.org/10.1002/cphy.c100088

Ruiz-Herrera, N., Guillén-Riquelme, A., Díaz-Román, A., \& Buela-Casal, G. (2021, January 07). Sleep, academic achievement, and cognitive performance in children with attention-deficit hyperactivity disorder: A polysomnographic study. Journal of Sleep Research. https://doi.org/10.1111/jsr.13275

Rzepka-Migut, B., \& Paprocka, J. (2020). Efficacy and Safety of Melatonin Treatment in Children with Autism Spectrum Disorder and Attention-Deficit/Hyperactivity Disorder-A Review of the Literature. Brain Sciences, 10(4), 219. MDPI AG. http://dx.doi.org/10.3390/brainsci10040219

Sanabra, M., Gómez-Hinojosa, T., Grau, N., \& Alda, J. A. (2021). Deficient Emotional Self-Regulation and Sleep Problems in ADHD with and without Pharmacological Treatment. Journal of Attention Disorders. https://doi.org/10.1177/1087054720986242

Staton, S., Rankin, P. S., Harding, M., Smith, S. S., Westwood, E., LeBourgeois, M. K., \& Thorpe, K. J. (2020). Many naps, one nap, none: A systematic review and meta-analysis of napping patterns in children 0-12 years. Sleep medicine reviews, 50, 101247. https://doi.org/10.1016/j.smrv.2019.101247

Suardiaz-Muro, M., Morante-Ruiz, M., Ortega-Moreno, M., Ruiz, M. A., Martín-Plasencia, P., \& Vela-Bueno, A. (2020). Sueño y rendimiento académico en estudiantes universitarios: revisión sistemática [Sleep and academic performance in university students: a systematic review]. Revista de neurologia, 71(2), 43-53. https://doi.org/10.33588/rn.7102.2020015

Sun, W., Ling, J., Zhu, X., Lee, T. M., \& Li, S. X. (2019). Associations of weekday-to-weekend sleep differences with academic performance and health-related outcomes in school-age children and youths. Sleep medicine reviews, 46, 27-53. https://doi.org/10.1016/j.smrv.2019.04.003

Yavuz-Kodat, E., Reynaud, E., Geoffray, M.-M., Limousin, N., Franco, P., Bonnet-Brilhault, F., Bourgin, P., \& Schroder, C. M. (2020). Disturbances of Continuous Sleep and Circadian Rhythms Account for Behavioral Difficulties in Children with Autism Spectrum Disorder. Journal of Clinical Medicine, 9(6), 1978. MDPI AG. Retrieved from http://dx.doi.org/10.3390/jcm9061978 